\title{
Modeling seasonal variation for mosquito-borne disease in the tropical monsoon environment
}

\author{
Ting-Ting Zheng ${ }^{1}$, Lin-Fei Nie ${ }^{*}\left(\mathbb{C}\right.$, Zhidong Teng ${ }^{1}$ and Yantao Luo'
}

"Correspondence: Ifnie@163.com

'College of Mathematics and

Systems Science, Xinjiang

University, Urumai, 830046,

P.R. China

\section{Springer}

\begin{abstract}
Mosquitoes play an important role in the spread of mosquito-borne diseases. Considering the sensitivity of mosquitoes' aquatic stage to the seasonal shift, in this paper, we present a seasonally forced mosquito-borne epidemic model by incorporating mosquitoes' aquatic stage (eggs, larvae, and pupae) and seasonal shift factor, which is a periodic discontinuous differential system. Firstly, some sufficient conditions for the existence and uniqueness of a disease-free solution are obtained. Further, we define the basic reproduction number $\mathcal{R}_{0}$, and obtain the stability of the disease-free solution when $\mathcal{R}_{0}$ is less than one. And, if $\mathcal{R}_{0}$ is greater than one, the mosquito-borne disease is uniformly persistent and the model admits a positive periodic solution. Finally, some numerical simulations are given to illustrate the main theoretical results. In addition, simulation results also imply that ignoring the effects of seasonal succession can overestimate or underestimate mosquito-borne disease trends.
\end{abstract}

Keywords: Mosquito-borne disease; Seasonal variation; Periodic solution; Stability and persistence

\section{Introduction}

In recent years, the spread of mosquito-borne diseases (such as dengue fever, malaria, Zika, chikungunya, yellow fever, and so on) has been characterized by high morbidity, high mortality, rapid growth in the number of cases, etc., which has become the major public health concern in the tropical and sub-tropical regions of the world. For example, in 2017, there were estimated 219 million cases of malaria in 87 countries, of which about 435,000 deaths [16]. According to the Philippine ministry of health, in 2019, more than 387,000 dengue cases (including 1452 deaths) have been registered in the Philippines as of November 2, up $99.3 \%$ from the same period last year [14].

Mathematical modeling is an important tool to understand the epidemiology of a mosquito-borne disease and assist in controlling the mosquito-borne disease. The first mosquito-borne disease model was developed to study the strategies to control malaria by Ross [21] in 1911. Subsequently, many scholars extended this model by considering acquired immunity in human population [5, 24], age structure in human population

(c) The Author(s) 2020. This article is licensed under a Creative Commons Attribution 4.0 International License, which permits use sharing, adaptation, distribution and reproduction in any medium or format, as long as you give appropriate credit to the original author(s) and the source, provide a link to the Creative Commons licence, and indicate if changes were made. The images or other third party material in this article are included in the article's Creative Commons licence, unless indicated otherwise in a credit line to the material. If material is not included in the article's Creative Commons licence and your intended use is not permitted by statutory regulation or exceeds the permitted use, you will need to obtain permission directly from the copyright holder. To view a copy of this licence, visit http://creativecommons.org/licenses/by/4.0/. 
$[27,29]$, spatial heterogeneity $[1,34]$, the incubation period of pathogen in the mosquito $[26,32,33]$, the life-cycle of mosquito [11, 12, 35], and so on. In addition, many kinds of dynamical models of mosquito-borne disease have been developed to put forward relevant strategies for disease control. We refer to some of them $[3,7,8]$ and the references therein.

It is well known that mosquito-borne diseases are influenced by complex demographic, environmental, and social factors. Temperature and rainfall are key environmental factors that determine the population size and the range of mosquitoes because they are critical to the length of mosquitoes' life cycle, the rate of reproduction and development of mosquitoes [20]. Therefore, many scholars have established periodic systems to study the effects of seasonality on mosquito-borne disease transmission (see $[9,17,31])$. Specifically, Mathieu et al. [4] established a vector-host model accounting for seasonal fluctuation in vector density and found that the periodic fluctuations of the vector population in phase with temperature variations suggest a strong climate effect on the vector density and, in turn, on the transmission of vector-borne. Lou et al. [15] presented and analyzed a mathematical malaria model with periodic environment. They obtained that dynamic behaviors of the model are determined by its basic reproduction number, and found that even small changes in temperature could change the prevalence of malaria.

To the best of our knowledge, in the existing models of seasonal mosquito-borne diseases, the effects of seasonality on the transmission of mosquito-borne disease are mainly characterized by a periodic continuous differential system. However, the periodic continuous differential system may not be appropriate for mosquito-borne disease modeling in regions with tropical monsoon climate, such as Mumbai (India), Colombo (Sri Lanka), Bangkok (Thailand), Ho Chi Minh City (Viet Nam), Phnom Penh (Cambodia), and so on mainly because there are only a dry season (Nov-Apr) and a rainy season (May-Oct) throughout the year in these areas, and the rainfall in the rainy season is significantly higher than that in the dry season. In fact, seasonal rainfall can increase the abundance of mosquitoes with aquatic larval stages, where reproduction depends on the availability of breeding sites [2]. Therefore, it is of great significance to consider a discontinuous periodic differential model to characterize the seasonal outbreak of mosquito-borne disease caused by seasonal change between rainy and dry seasons.

Based on the above discussion, in this paper, we propose a mosquito-borne disease transmission model with seasonal variation, which is a periodic discontinuous differential system. Applying the calculation method of the basic reproduction number for the continuous periodic differential system given in Wang and Zhao [30] and the theoretical analysis method of the discontinuous periodic differential system given in Tang et al. [23], we calculate the basic reproduction number for this model and discuss the uniform persistence of the disease and threshold dynamics. The organization of this paper is as follows. In the next section, model formulation, some hypotheses, and the well-posedness of this model are given. In Sect. 3, the basic reproduction number is calculated, and we show that it is the threshold value for uniform persistence and global extinction of the disease. In Sect. 4, we give some numerical simulations and discussion to illustrate our theoretical results, and we give some concluding remarks in the last section.

\section{Model formulation and preliminaries}

According to the mosquito-borne epidemic compartmental models in previous studies $[22,28]$, the total human population at time $t$, denoted by $N_{h}(t)$, is divided into three 
classical classes: the susceptible $S_{h}(t)$, the infected $I_{h}(t)$, and the recovered $R_{h}(t)$. For the mosquito population, we consider the aquatic mosquitoes (comprising eggs, larvae, and pupae) and adult mosquitoes, where the adult population at time $t$, denoted by $N_{m}(t)$, is split into two classes: susceptible mosquitoes $S_{m}(t)$ and infected mosquitoes $I_{m}(t)$, the aquatic population at time $t$ is denoted by $A(t)$. Further, the following basic assumptions are introduced for our model.

$\left(H_{1}\right)$ Considering the influence of seasonal factors (such as humidity, temperature, etc.) on mosquitoes' reproduction rate and breeding sites, we assume that the recruitment rate of the aquatic mosquitoes is governed by a logistic equation, in which these coefficients are periodic functions on account of seasonal effects.

$\left(H_{2}\right)$ For human population, let $\Lambda_{h}, \mu_{h}, d_{h}, \gamma$, and $\eta$ be the human recruitment rate, natural death rate, disease induced death rate, recovery rate, and lose immunity rate, respectively. Denote the seasonal forced transmission coefficient from infected mosquitoes to a susceptible human by $\beta(t)$, which is affected by seasonal succession.

$\left(H_{3}\right)$ For mosquito population, let $k(t), b(t), \mu_{a}(t)$, and $\mu_{m}(t)$ represent the carrying capacity of aquatic mosquitoes, mosquito birth rate, aquatic mosquito death rate, and adult mosquito death rate, respectively. The per capita rate of maturation from the aquatic form to the adult one is denoted by $\phi(t)$. Let $\alpha(t)$ denote the transmission coefficient from the infected human to susceptible mosquitoes.

$\left(H_{4}\right)$ Let $\omega$ be given as the period of disease transmission. Let $J_{1}$ and $J_{2}$ denote the dry season and the rainy season, respectively, where $J_{1}=[n \omega, n \omega+\theta \omega)$ and $J_{2}=[n \omega+$ $\theta \omega,(n+1) \omega)$. Here, $n \in \mathbb{Z}_{+}$and $\theta \in(0,1)$ is the fraction of the rainy season to the whole infection cycle.

According to the assumptions above and taking into account the interaction between human and mosquito population, we give the following ordinary differential equations:

$$
\left\{\begin{array}{l}
\frac{\mathrm{d} S_{h}(t)}{\mathrm{d} t}=\Lambda_{h}+\eta R_{h}(t)-\beta(t) S_{h}(t) I_{m}(t)-\mu_{h} S_{h}(t), \\
\frac{\mathrm{d} I_{h}(t)}{\mathrm{d} t}=\beta(t) S_{h}(t) I_{m}(t)-\left(\gamma_{h}+\mu_{h}+d_{h}\right) I_{h}(t), \\
\frac{\mathrm{d} R_{h}(t)}{\mathrm{d} t}=\gamma_{h} I_{h}(t)-\left(\mu_{h}+\eta\right) R_{h}(t), \\
\frac{\mathrm{d}(t)}{\mathrm{d} t}=b(t) N_{m}(t)\left(1-\frac{A(t)}{k(t)}\right)-\phi(t) A(t)-\mu_{a}(t) A(t), \\
\frac{\mathrm{d} S_{m}(t)}{\mathrm{d} t}=\phi(t) A(t)-\alpha(t) S_{m}(t) I_{h}(t)-\mu_{m}(t) S_{m}(t), \\
\frac{\mathrm{d} I_{m}(t)}{\mathrm{d} t}=\alpha(t) S_{m}(t) I_{h}(t)-\mu_{m}(t) I_{m}(t),
\end{array}\right.
$$

with the following piecewise constant functions:

$$
\begin{aligned}
& \beta(t)=\left\{\begin{array}{ll}
\beta_{1}, & t \in J_{1}, \\
\beta_{2}, & t \in J_{2},
\end{array} \quad b(t)= \begin{cases}b_{1}, & t \in J_{1}, \\
b_{2}, & t \in J_{2},\end{cases} \right. \\
& k(t)=\left\{\begin{array}{ll}
k_{1}, & t \in J_{1}, \\
k_{2}, & t \in J_{2},
\end{array} \quad \phi(t)= \begin{cases}\phi_{1}, & t \in J_{1}, \\
\phi_{2}, & t \in J_{2},\end{cases} \right.
\end{aligned}
$$

and

$$
\mu_{a}(t)=\left\{\begin{array}{ll}
\mu_{a 1}, & t \in J_{1}, \\
\mu_{a 2}, & t \in J_{2},
\end{array} \quad \alpha(t)=\left\{\begin{array}{ll}
\alpha_{1}, & t \in J_{1}, \\
\alpha_{2}, & t \in J_{2},
\end{array} \quad \mu_{m}(t)= \begin{cases}\mu_{m 1}, & t \in J_{1}, \\
\mu_{m 2}, & t \in J_{2},\end{cases}\right.\right.
$$


where $J_{1}=[n \omega, n \omega+\theta \omega)$ and $J_{2}=[n \omega+\theta \omega,(n+1) \omega)$. Assume $\beta_{1} \geq \beta_{2}>0, b_{1} \geq b_{2}>0$, $k_{1} \geq k_{2}>0, \phi_{1} \geq \phi_{2}>0, \mu_{a 2} \geq \mu_{a 1}>0, \alpha_{1} \geq \alpha_{2}>0$, and $\mu_{m 1} \geq \mu_{m 2}>0$.

In view of the biological background of model (1), we only consider the solution of model (1) with initial conditions $\left(S_{h}(0), I_{h}(0), R_{h}(0), A(0), S_{m}(0), I_{m}(0)\right) \in \mathbb{R}_{+}^{6}=\left\{\left(x_{1}, \ldots, x_{6}\right): x_{i} \geq\right.$ $0, i=1, \ldots, 6\}$.

Now, we can give the following results about the existence, uniqueness, and boundedness of solutions of model (1).

Theorem 1 For any initial value condition $\left(S_{h}(0), I_{h}(0), R_{h}(0), A(0), S_{m}(0), I_{m}(0)\right) \in \mathbb{R}_{+}^{6}$, model (1) has a unique global nonnegative solution $\left(S_{h}(t), I_{h}(t), R_{h}(t), A(t), S_{m}(t), I_{m}(t)\right)$ for all $t \geq 0$, and this solution is ultimately bounded.

The global existence and uniqueness of the solution can be followed by Theorem 2.1 in [23]. The positivity and boundedness can be easily obtained, here omitted.

Consider the following two disease-free subsystems of model (1):

$$
\frac{\mathrm{d} S_{h}(t)}{\mathrm{d} t}=\Lambda_{h}-\mu_{h} S_{h}(t)
$$

and

$$
\left\{\begin{array}{l}
\frac{\mathrm{d} A(t)}{\mathrm{d} t}=b(t) S_{m}(t)\left(1-\frac{A(t)}{k(t)}\right)-\left(\phi(t)+\mu_{a}(t)\right) A(t), \\
\frac{\mathrm{d} S_{m}(t)}{\mathrm{d} t}=\phi(t) A(t)-\mu_{m}(t) S_{m}(t) .
\end{array}\right.
$$

On the global dynamics of models (2) and (3), we have the following lemmas.

Lemma 1 Model (2) has a unique positive globally asymptotically stable equilibrium $S_{h}^{*}(t)=\Lambda_{h} / \mu_{h}$.

The proof of Lemma 1 is obvious, so it is omitted.

Lemma 2 If $\phi_{2} b_{2}>\left(\phi_{1}+\mu_{a 1}\right) \mu_{m 1}$, then model (3) is persistent and has a positive $\omega$-periodic solution $\left(A^{*}(t), S_{m}^{*}(t)\right)$. Further, the positive $\omega$-periodic solution $\left(A^{*}(t), S_{m}^{*}(t)\right)$ is globally uniformly attractive when $\liminf _{t \rightarrow \infty} \Theta(t)>0$, where

$$
\Theta(t)=\mu_{m}(t)-b(t)\left|1-\frac{A^{*}(t)}{k(t)}\right| .
$$

Proof By the similarly method of Theorem 3 and Theorem 4 given in Ref. [10], it can be obtained that when $\phi_{2} b_{2}>\left(\phi_{1}+\mu_{a 1}\right) \mu_{m 1}$, model (3) is persistent and has one positive periodic solution $\left(A^{*}(t), S_{m}^{*}(t)\right)$. Now, we will prove global uniform attractiveness of this periodic solution.

Let $\left(A(t), S_{m}(t)\right)$ be any positive solution of model (3). Defining a function $V(t)=\mid A(t)-$ $A^{*}(t)|+| S_{m}(t)-S_{m}^{*}(t) \mid$ and calculating the upper right derivative of $V(t)$ along the solutions of model (3), it follows that

$$
\begin{aligned}
D^{+} V(t)= & \operatorname{sign}\left(A(t)-A^{*}(t)\right)\left\{b(t)\left(S_{m}(t)-S_{m}^{*}(t)\right)-\left(\phi(t)+\mu_{a}(t)\right)\left(A(t)-A^{*}(t)\right)\right. \\
& \left.-\frac{b(t)}{k(t)}\left[S_{m}(t)\left(A(t)-A^{*}(t)\right)+A^{*}(t)\left(S_{m}(t)-S_{m}^{*}(t)\right)\right]\right\}
\end{aligned}
$$




$$
\begin{aligned}
& +\operatorname{sign}\left(S_{m}(t)-S_{m}^{*}(t)\right)\left[\phi(t)\left(A(t)-A^{*}(t)\right)-\mu_{m}(t)\left(S_{m}(t)-S_{m}^{*}(t)\right)\right] \\
\leq & \left\{b(t)-\frac{b(t)}{k(t)} A^{*}(t)\right\} \operatorname{sign}\left(A(t)-A^{*}(t)\right)\left(S_{m}(t)-S_{m}^{*}(t)\right) \\
& -\left\{\frac{b(t)}{k(t)} S_{m}(t)+\mu_{a}(t)\right\}\left|A(t)-A^{*}(t)\right|-\mu_{m}(t)\left|S_{m}(t)-S_{m}^{*}(t)\right| \\
\leq & -\Theta(t)\left|S_{m}(t)-S_{m}^{*}(t)\right|-\left\{\frac{b(t)}{k(t)} S_{m}(t)+\mu_{a}(t)\right\}\left|A(t)-A^{*}(t)\right| .
\end{aligned}
$$

Obviously, there exist positive constants $\varrho_{1}, \varrho_{2}$ and $T_{1} \geq 0$ such that

$$
\Theta(t) \geq \varrho_{1}>0, \quad \frac{b(t)}{k(t)} S_{m}(t)+\mu_{a}(t) \geq \varrho_{2}>0 \quad \text { for all } t \geq T_{1} .
$$

Integrating both sides of the above inequality on interval $\left[T_{1}, t\right]$ yields that

$$
\begin{aligned}
V\left(T_{1}\right) & \geq V(t)+\int_{T_{1}}^{t}\left\{\Theta(s)\left|S_{m}(s)-S_{m}^{*}(s)\right|+\left(\frac{b(s)}{k(s)} S_{m}(s)+\mu_{a}(s)\right)\left|A(s)-A^{*}(s)\right|\right\} \mathrm{d} s \\
& \geq V(t)+\int_{T_{1}}^{t}\left\{\varrho_{1}\left|S_{m}(s)-S_{m}^{*}(s)\right|+\varrho_{2}\left|A(s)-A^{*}(s)\right|\right\} \mathrm{d} s .
\end{aligned}
$$

Therefore, $V(t)$ is bounded on $\left[T_{1}, \infty\right]$ and

$$
\int_{T_{1}}^{\infty}\left|A(s)-A^{*}(s)\right| \mathrm{d} s<\infty, \quad \int_{T_{1}}^{\infty}\left|S_{m}(s)-S_{m}^{*}(s)\right| \mathrm{d} s<\infty .
$$

By Theorem 1, $\left|A(s)-A^{*}(s)\right|$ and $\left|S_{m}(s)-S_{m}^{*}(s)\right|$ are bounded on $\left[T_{1}, \infty\right]$. On the other hand, it is easy to see that $\mathrm{d} A(t) / \mathrm{d} t, \mathrm{~d} A^{*}(t) / \mathrm{d} t, \mathrm{~d} S_{m}(t) / \mathrm{d} t$, and $\mathrm{d} S_{m}^{*}(t) / \mathrm{d} t$ are bounded for $t \geq T_{1}$. Therefore, $\left|A(s)-A^{*}(s)\right|$ and $\left|S_{m}(s)-S_{m}^{*}(s)\right|$ are uniformly continuous on $\left[T_{1}, \infty\right]$. By Barbalat's lemma [13], we conclude that $\lim _{t \rightarrow \infty}\left|A(t)-A^{*}(t)\right|=0, \lim _{t \rightarrow \infty}\left|S_{m}(t)-S_{m}^{*}(t)\right|=$ 0 . This completes the proof.

As consequence of Lemmas 1 and 2, we can obtain that if $\phi_{2} b_{2}>\mu_{m 1}\left(\phi_{1}+\mu_{a 1}\right)$ and $\liminf _{t \rightarrow \infty} \Theta(t)>0$, then model (1) has a unique solution $\left(S_{h}^{*}(t), 0,0, A^{*}(t), S_{m}^{*}(t), 0\right)$, which implies that the disease is extinct.

\section{Basic reproduction number and threshold dynamics}

In this section, we introduce the threshold quantity for the piecewise periodic model (1) and analyze its properties. First, we need to define the next infection operator for model (1), which arises from the combination with the idea in Ref. [30] for periodic ordinary differential models.

$$
\text { Let } x(t)=\left(I_{h}(t), I_{m}(t), S_{h}(t), R_{h}(t), A(t), S_{m}(t)\right)^{T}, x^{*}(t)=\left(0,0, S_{h}^{*}(t), 0, A^{*}(t), S_{m}^{*}(t)\right) \text { and }
$$

$$
\mathcal{F}_{i}(t, x)=\left(\begin{array}{c}
\beta_{i} S_{h} I_{m} \\
\alpha_{i} S_{m} I_{h} \\
0 \\
0 \\
0 \\
0
\end{array}\right), \quad \mathcal{V}_{i}(t, x)=\left(\begin{array}{c}
\left(\gamma_{h}+\mu_{h}+d_{h}\right) I_{h} \\
\mu_{m i} I_{m} \\
-\Lambda_{h}-\eta R_{h}+\beta_{i} S_{h} I_{m}+\mu_{h} S_{h} \\
-\gamma_{h} I_{h}+\left(\eta+\mu_{h}\right) R_{h} \\
-b_{i} N_{m}\left(1-\frac{A}{k_{i}}\right)+\left(\phi_{i}+\mu_{a i}\right) A \\
-\phi_{i} A+\alpha_{i} S_{m} I_{h}+\mu_{m i} S_{m}
\end{array}\right), \quad i=1,2 .
$$


Then model (1) can be rewritten as

$$
\frac{\mathrm{d} x}{\mathrm{~d} t}=\mathcal{F}(t, x)-\mathcal{V}(t, x)
$$

where $\mathcal{F}(t, x)=\mathcal{I}_{J_{1}} \mathcal{F}_{1}(t, x)+\mathcal{I}_{J_{2}} \mathcal{F}_{2}(t, x), \mathcal{V}(t, x)=\mathcal{I}_{J_{1}} \mathcal{V}_{1}(t, x)+\mathcal{I}_{J_{2}} \mathcal{V}_{2}(t, x)$, and

$$
\mathcal{I}_{J_{i}}= \begin{cases}1, & \text { as } t \in J_{i}, \\ 0, & \text { as } t \notin J_{i} .\end{cases}
$$

Denote

$$
F_{i}(t)=\left(\frac{\partial \mathcal{F}_{i j}\left(t, x^{*}(t)\right)}{\partial x_{k}}\right)_{1 \leq i, j, k \leq 2}, \quad V_{i}(t)=\left(\frac{\partial \mathcal{V}_{i j}\left(t, x^{*}(t)\right)}{\partial x_{k}}\right)_{1 \leq i, j, k \leq 2},
$$

where $\mathcal{F}_{i j}(t, x(t))$ and $\mathcal{V}_{i j}(t, x(t))$ are the $j$ th components of $\mathcal{F}_{i}(t, x(t))$ and $\mathcal{V}_{i}(t, x(t))$, respectively. Then, by simple computations, it follows that

$$
F_{i}(t)=\left(\begin{array}{cc}
0 & \beta_{i} \Lambda_{h} / \mu_{h} \\
\alpha_{i} S_{m}^{*}(t) & 0
\end{array}\right) \quad \text { and } \quad V_{i}(t)=\left(\begin{array}{cc}
\gamma_{h}+\mu_{h}+d_{h} & 0 \\
0 & \mu_{m i}
\end{array}\right), \quad i=1,2 .
$$

Now, we extend $t \in \mathbb{R}_{+}$to $t \in \mathbb{R}$ and introduce some new notations. When $t \in \bigcup_{n=-\infty}^{+\infty}\left(J_{1} \cup\right.$ $\left.J_{2}\right)=(-\infty,+\infty)$ and

$$
\begin{aligned}
& F(t)=\mathcal{I}_{J_{1}} F_{1}(t)+\mathcal{I}_{J_{2}} F_{2}(t)=\left(\begin{array}{cc}
0 & \beta(t) \Lambda_{h} / \mu_{h} \\
\alpha(t) S_{m}^{*}(t) & 0
\end{array}\right), \\
& V(t)=\mathcal{I}_{J_{1}} V_{1}(t)+\mathcal{I}_{J_{2}} V_{2}(t)=\left(\begin{array}{cc}
\gamma_{h}+\mu_{h}+d_{h} & 0 \\
0 & \mu_{m}(t)
\end{array}\right) .
\end{aligned}
$$

Obviously, $F$ and $V$ are $2 \times 2$ piecewise continuous periodic matrices with period $\omega$ in $\mathbb{R}$, and $-V(t)$ is cooperative.

Let $\mathbb{Y}(t, s), t \geq s$, be the evolution operator of the linear system

$$
\frac{\mathrm{d} y}{\mathrm{~d} t}=-V(t) y \text {. }
$$

Then the $2 \times 2$ matrix $\mathbb{Y}(t, s)$ satisfies

$$
\frac{\mathrm{d} \mathbb{Y}(t, s)}{\mathrm{d} t}=-V(t) \mathbb{Y}(t, s), \quad \forall t \geq s, s \in \mathbb{R}, \quad \mathbb{Y}(s, s)=\mathrm{E},
$$

where $\mathrm{E}$ is the $2 \times 2$ identity matrix. Therefore, the monodromy matrix $\Phi_{-V}(t)$ of model (6) equals $\mathbb{Y}(t, 0), t \geq 0$. According to Remark 3.5 in Sect. III.7 of Ref. [6], there exist $K_{1}>0$ and $K_{2}>0$ such that

$$
\|\mathbb{Y}(t, s)\| \leq K_{1} \mathrm{e}^{-K_{2}(t-s)}, \quad \forall t \geq s, s \in \mathbb{R} .
$$

Obviously, there exists a constant $K_{3}>0$ such that $\|F(t)\|<K_{3}$ followed by the boundedness of $F(t)$. Therefore,

$$
\|\mathbb{Y}(t, t-a) F(t, t-a)\| \leq K_{1} K_{3} \mathrm{e}^{-K_{2} a}, \quad \forall t \in \mathbb{R}, a \in[0,+\infty)
$$


Let $\mathbb{C}_{\omega}$ be the ordered Banach space of all $\omega$-periodic continuous functions from $\mathbb{R}$ to $\mathbb{R}^{2}$ with the maximum norm $\|\cdot\|_{c}$, and $\mathbb{C}_{\omega}^{+}=\left\{\mathbb{I} \in \mathbb{C}_{\omega}: \mathbb{I}(s) \geq 0, s \in \mathbb{R}\right\}$. Assume that $\mathbb{I}(s) \in \mathbb{C}_{\omega}^{+}$is the initial distribution of infectious individuals in this periodic environment, then $F(s) \mathbb{I}(s)$ is the rate of new infectious produced by the infected individuals who were introduced at time $s$, and $\mathbb{Y}(t, s) F(s) \mathbb{I}(s)$ represents the distributions of those infected individuals who were newly infected at time $s$ and remain in the infected compartment at time $t$ for $t \geq s$. It follows that

$$
\int_{-\infty}^{t} \mathbb{Y}(t, s) F(s) \mathbb{I}(s) \mathrm{d} s=\int_{0}^{\infty} \mathbb{Y}(t, t-a) F(t-a) \mathbb{I}(t-a) \mathrm{d} a,
$$

which denotes the distribution of accumulative new infections at time $t$ produced by all those infected individuals $\mathbb{I}(s)$ introduced at previous time to $t$. Define a linear operator $\mathcal{L}: \mathbb{C}_{\omega} \rightarrow \mathbb{C}_{\omega}$ by

$$
(\mathcal{L} \mathbb{I})(t)=\int_{-\infty}^{t} \mathbb{Y}(t, s) F(s) \mathbb{I}(s) \mathrm{d} s=\int_{0}^{\infty} \mathbb{Y}(t, t-a) F(t-a) \mathbb{I}(t-a) \mathrm{d} a
$$

Similar to Lemma 3.1 given in Ref. [23], the linear operator $\mathcal{L}$ is well defined.

Lemma 3 The operator $\mathcal{L}$ is positive, continuous, and compact on $\mathbb{C}_{\omega}$.

Therefore, following Wang and Zhao in [30], we call $\mathcal{L}$ the next infection operator and define the basic reproduction number $\mathcal{R}_{0}$ for model (1) by

$$
\mathcal{R}_{0}=\rho(\mathcal{L}),
$$

where $\rho(\mathcal{L})$ is the spectral radius of $\mathcal{L}$. Similar to the analysis of Ref. [23], it is easy to follow the argument in [29] to characterize $\mathcal{R}_{0}$. Set $U_{\lambda}(t, s)(t \geq s, s \in \mathbb{R})$ be the evolution operator of the following auxiliary $\omega$-periodic switching model:

$$
\frac{\mathrm{d} U(t)}{\mathrm{d} t}=\left\{-V(t)+\frac{F(t)}{\lambda}\right\} U(t), \quad \lambda \in(0,+\infty) .
$$

Then $U(\omega, 0, \lambda)=\Phi_{F-V}(\omega)$. Following the ideas in Ref. [30], we have the following result.

\section{Lemma 4}

(i) If $\rho\left(\Phi_{F-V}(\omega)\right)=1$ has a positive solution $\lambda_{0}$, then $\lambda_{0}$ is an eigenvalue of $\mathcal{L}$, and so $\mathcal{R}_{0}>0$.

(ii) If $\mathcal{R}_{0}>0$, then $\lambda=\mathcal{R}_{0}$ is the unique solution of $\rho\left(\Phi_{F-V}(\omega)\right)=1$.

(iii) $\mathcal{R}_{0}>0$ if and only if $\rho\left(\Phi_{F-V}(\omega)\right)<1$ for all $\lambda>0$.

Applying Lemma 4 directly, we can obtain the following result on basic reproduction number $\mathcal{R}_{0}$.

\section{Theorem 2}

(a) $\mathcal{R}_{0}=1$ if and only if $\rho\left(\Phi_{F-V}(\omega)\right)=1$;

(b) $\mathcal{R}_{0}>1$ if and only if $\rho\left(\Phi_{F-V}(\omega)\right)>1$;

(c) $\mathcal{R}_{0}<1$ if and only if $\rho\left(\Phi_{F-V}(\omega)\right)<1$. 
Furthermore, the disease-free solution $\left(S_{h}^{*}(t), 0,0, A^{*}(t), S_{m}^{*}(t), 0\right)$ is locally asymptotically stable if $\mathcal{R}_{0}<1$ and unstable if $\mathcal{R}_{0}>1$.

Next, we explore the threshold condition, which determines the extinction and persistence of the disease.

Theorem 3 If $\mathcal{R}_{0}<1, \phi_{2} b_{2}>\mu_{m 1}\left(\phi_{1}+\mu_{a 1}\right)$, and $\liminf _{t \rightarrow \infty} \Theta(t)>0$, then solution $\left(S_{h}^{*}(t), 0,0, A^{*}(t), S_{m}^{*}(t), 0\right)$ is globally asymptotically stable, where $\Theta(t)$ is given by (4).

Proof From Theorem 2, we obtain that if $\mathcal{R}_{0}<1$, solution $\left(S_{h}^{*}(t), 0,0, A^{*}(t), S_{m}^{*}(t), 0\right)$ is locally asymptotically stable. Now, it suffices to prove the global attractiveness of solution $\left(S_{h}^{*}(t), 0,0, A^{*}(t), S_{m}^{*}(t), 0\right)$ for $\mathcal{R}_{0}<1$. It follows from conclusion (c) of Theorem 2 that we can choose a small enough constant $\epsilon>0$ such that $\rho\left(\Phi_{F-V+\epsilon N}(\omega)\right)<1$, where

$$
N(t)=\left(\begin{array}{cc}
0 & \beta(t) \\
\alpha(t) & 0
\end{array}\right) .
$$

It can be shown, by Lemma 1 and Lemma 2, that if $\phi_{2} b_{2}>\mu_{m 1}\left(\phi_{1}+\mu_{a 1}\right)$ and $\liminf _{t \rightarrow \infty} \Theta(t)>0$, then there exists $t_{1}>0$ such that

$$
S_{h}(t) \leq \Lambda_{h} / \mu_{h}+\epsilon \quad \text { and } \quad S_{m}(t) \leq S_{m}^{*}(t)+\epsilon \quad \text { for all } t>t_{1} .
$$

Substituting the inequalities in (7) into model (1), we have

$$
\left\{\begin{array}{l}
\frac{\mathrm{d} I_{h}(t)}{\mathrm{d} t} \leq \beta(t)\left(\Lambda_{h} / \mu_{h}+\epsilon\right) I_{m}(t)-\left(\gamma_{h}+\mu_{h}+d_{h}\right) I_{h}(t), \\
\frac{\mathrm{d} I_{m}(t)}{\mathrm{d} t} \leq \alpha(t)\left(S_{m}^{*}(t)+\epsilon\right) I_{h}(t)-\mu_{m}(t) I_{m}(t)
\end{array}\right.
$$

for all $t>t_{1}$. Consider the following auxiliary system:

$$
\left\{\begin{array}{l}
\frac{\widetilde{\mathrm{d}}_{h}(t)}{\mathrm{d} t}=\beta(t)\left(\Lambda_{h} / \mu_{h}+\epsilon\right) \widetilde{I}_{m}(t)-\left(\gamma_{h}+\mu_{h}+d_{h}\right) \widetilde{I}(t), \\
\frac{\mathrm{d} \widetilde{I}_{m}(t)}{\mathrm{d} t}=\alpha(t)\left(S_{m}^{*}(t)+\epsilon\right) \widetilde{I}_{h}(t)-\mu_{m}(t) \widetilde{I}_{m}(t)
\end{array}\right.
$$

which is a periodic linear discontinuous model with period $\omega$. For the convenience, we will rewrite it as follows:

$$
\frac{\mathrm{d}}{\mathrm{d} t}\left(\begin{array}{c}
\widetilde{I}_{h}(t) \\
\widetilde{I}_{m}(t)
\end{array}\right)=(F(t)-V(t)-\epsilon N(t))\left(\begin{array}{c}
\widetilde{I}_{h}(t) \\
\widetilde{I}_{m}(t)
\end{array}\right) .
$$

When $\mathcal{R}_{0}<1$, we have $\rho\left(\Phi_{F-V+\epsilon N}(\omega)\right)<1$, which implies that $(0,0)$ is a globally asymptotically stable solution of model (8). By the comparison principle, we have $\lim _{t \rightarrow \infty}\left(I_{h}(t), I_{m}(t)\right)=(0,0)$. By the equations of $S_{h}, R_{h}, A$, and $S_{m}$ of model (1), Lemmas 1 and 2, we also obtain $\lim _{t \rightarrow \infty}\left(S_{h}(t), R_{h}(t), A(t), S_{m}(t)\right)=\left(S_{h}^{*}(t), 0, A^{*}(t), S_{m}^{*}(t)\right)$. Hence, the disease-free solution $\left(S^{*}(t), 0,0, A^{*}(t), S_{m}^{*}(t), 0\right)$ is globally attractive. Therefore, solution $\left(S_{h}^{*}(t), 0,0, A^{*}(t), S_{m}^{*}(t), 0\right)$ of model (1) is globally asymptotically stable.

It follows from an epidemiological concept that if $\mathcal{R}_{0}<1$ the number of infected human and infectious mosquitoes tends to zero with the increase of time and the disease is extinct. However, the disease-free periodic solution is unstable, that is, no matter how small 
the number of infected hosts or infectious vectors in the initial state, the disease will occur repeatedly in the region and become endemic due to its long-term prevalence. In the following, we show that when $\mathcal{R}_{0}>1$, the disease is uniformly persistent.

Theorem 4 If $\mathcal{R}_{0}>1, \phi_{2} b_{2}>\mu_{m 1}\left(\phi_{1}+\mu_{a 1}\right)$ and $\liminf _{t \rightarrow \infty} \Theta(t)>0$, then there exists a constant $\vartheta>0$ such that every solution $\left(S_{h}(t), I_{h}(t), R_{h}(t), A(t), S_{m}(t), I_{m}(t)\right)$ of model (1) satisfies

$$
\liminf _{t \rightarrow+\infty} I_{h}(t) \geq \vartheta, \quad \liminf _{t \rightarrow+\infty} I_{m}(t) \geq \vartheta .
$$

Proof Define

$$
\begin{aligned}
& \mathbb{X}=\left\{\left(S_{h}, I_{h}, R_{h}, A, S_{m}, I_{m}\right): S_{h}>0, I_{h} \geq 0, R_{h} \geq 0, A>0, S_{m}>0, I_{m} \geq 0\right\}, \\
& \mathbb{X}_{0}=\left\{\left(S_{h}, I_{h}, R_{h}, A, S_{m}, I_{m}\right) \in \mathbb{X}: I_{h}>0, I_{m}>0\right\},
\end{aligned}
$$

and

$$
\partial \mathbb{X}_{0}=\mathbb{X} \backslash \mathbb{X}_{0}=\left\{\left(S_{h}, I_{h}, R_{h}, A, S_{m}, I_{m}\right) \in \mathbb{X}: I_{h} I_{m}=0\right\}
$$

From model (1), it is easy to see that $\mathbb{X}$ and $\mathbb{X}_{0}$ are positively invariant and $\partial \mathbb{X}_{0}$ is also a relatively closed set in $\mathbb{X}$.

Let $\mathcal{P}: \mathbb{X} \rightarrow \mathbb{X}$ be the Poincaré map associated with model (1), that is,

$$
\mathcal{P}\left(P_{0}\right)=u\left(\omega, P_{0}\right)=u_{2}\left(\omega, \theta \omega, u_{1}\left(\theta \omega, 0, P_{0}\right)\right)
$$

where $u\left(t, P_{0}\right)$ is a solution of model (1) under the initial value $P_{0} \in \mathbb{R}_{+}^{5}$, and $u_{i}\left(t, t_{*}, P_{*}\right)$ $(i=1,2)$ is the solution semi-flow of the following model:

$$
\left\{\begin{array}{l}
\frac{\mathrm{d} S_{h}(t)}{\mathrm{d} t}=\Lambda_{h}+\eta R_{h}(t)-\beta_{i} S_{h}(t) I_{m}(t)-\mu_{h} S_{h}(t), \\
\frac{\mathrm{d} d_{h}(t)}{\mathrm{d} t}=\beta_{i} S_{h}(t) I_{m}(t)-\left(\gamma_{h}+\mu_{h}+d_{h}\right) I_{h}(t), \\
\frac{\mathrm{d} R_{h}(t)}{\mathrm{d} t}=\gamma_{h} I(t)-\left(\mu_{h}+\eta\right) R_{h}(t) \\
\frac{\mathrm{d}(t)}{\mathrm{d} t}=b_{i} N_{m}(t)\left(1-\frac{A(t)}{k_{i}}\right)-\left(\phi_{i}+\mu_{a i}\right) A(t), \\
\frac{\mathrm{d} S_{m}(t)}{\mathrm{d} t}=\phi_{i} A(t)-\alpha_{i} S_{m}(t) I_{h}(t)-\mu_{m i} S_{m}(t) \\
\frac{\mathrm{d}{ }_{m}(t)}{\mathrm{d} t}=\alpha_{i} S_{m}(t) I_{h}(t)-\mu_{m i} I_{m}(t) \\
u_{i}\left(t_{*}, t_{*}, P_{*}\right)=P_{*}, \quad P_{*} \in \mathbb{R}_{+}^{5} .
\end{array}\right.
$$

It follows from the continuity of solution of model (1) with respect to the initial value $P_{0}$ that $\mathcal{P}$ is compact. Moreover, by Theorem 1, we obtain that $\mathcal{P}$ is point dissipative on $\mathbb{X}$.

Define

$$
M_{\partial}=\left\{P_{0} \in \partial \mathbb{X}_{0}: \mathcal{P}^{k}\left(P_{0}\right) \in \partial \mathbb{X}_{0}, \forall k \geq 0\right\}
$$

It is a positive invariant set of $\mathcal{P}$ in $\partial \mathbb{X}_{0}$. Now, we claim

$$
M_{\partial}=\left\{\left(S_{h}, 0,0, A, S_{m}, 0\right): S_{h}>0, A>0, S_{m}>0\right\} .
$$


In fact, $\left\{\left(S_{h}, 0,0, A, S_{m}, 0\right): S_{h}>0, A>0, S_{m}>0\right\} \subset M_{\partial}$. On the other hand, if $M_{\partial} \backslash$ $\left\{\left(S_{h}, 0,0, A, S_{m}, 0\right): S_{h}>0, A>0, S_{m}>0\right\} \neq \emptyset$, that is, there exists at least a point $\left(S_{h 0}, I_{h 0}, R_{h 0}\right.$, $\left.A_{0}, S_{m 0}, I_{m 0}\right) \in M_{\partial}$ satisfying $I_{h 0}>0$ or $I_{m 0}>0$. If $I_{h 0}=0$ and $I_{m 0}>0$, then

$$
I_{m}(t) \geq I_{m 0} \mathrm{e}^{-\mu_{2} t}>0 \quad \text { for all } t>0 .
$$

Because $S_{h 0}>0$, it can be shown from the first equation of model (1) that $S_{h}(t)>0$ for all $t>0$. Hence,

$$
I_{h}(t)=\mathrm{e}^{-\left(\gamma_{h}+\mu_{h}+d_{h}\right) s}\left[I_{h 0}+\int_{0}^{t} \beta(s) S_{h}(s) I_{m}(s) \mathrm{e}^{\left(\gamma_{h}+\mu_{h}+d_{h}\right) s}\right]>0 \quad \text { for all } t>0 .
$$

Similarly, if $I_{h 0}>0$ and $I_{m 0}=0$, then $I_{h}(t)>0$ and $I_{m}(t)>0$ for all $t>0$.

This shows that $\left(S_{h}(t), I_{h}(t), R_{h}(t), A(t), S_{m}(t), I_{m}(t)\right) \notin \partial \mathbb{X}_{0}$. Hence, $\left(S_{h 0}, I_{h 0}, R_{h 0}, A_{0}, S_{m 0}\right.$, $\left.I_{m 0}\right) \notin M_{\partial}$, which leads to a contradiction. It indicates that $M_{\partial} \subseteq\left\{\left(S_{h}, 0,0, A, S_{m}, 0\right): S_{h}>\right.$ $\left.0, A>0, S_{m}>0\right\}$. Hence, (11) is proved. In $M_{\partial}$, model (1) degenerates to

$$
\left\{\begin{array}{l}
\frac{\mathrm{d} S_{h}(t)}{\mathrm{d} t}=\Lambda_{h}-\mu_{h} S_{h}(t), \\
\frac{\mathrm{d} A(t)}{\mathrm{d} t}=b(t) S_{m}(t)\left(1-\frac{A(t)}{k(t)}\right)-\left(\phi(t)+\mu_{a}(t)\right) A(t), \\
\frac{\mathrm{d} S_{m}(t)}{\mathrm{d} t}=\phi(t) A(t)-\mu_{m}(t) S_{m}(t) .
\end{array}\right.
$$

Clearly, if $\phi_{2} b_{2}>\mu_{m 1}\left(\phi_{1}+\mu_{a 1}\right)$ and $\liminf _{t \rightarrow \infty} \Theta(t)>0$, model (12) has a globally stable $\omega$-periodic solution $\left(\Lambda_{h} / \mu_{h}, A^{*}(t), S_{m}^{*}(t)\right)$. Therefore, the map $\mathcal{P}$ has a unique globally attractive fixed point restricted in $M_{\partial}$, which is $M_{1}=\left(\Lambda_{h} / \mu_{h}, 0,0, A^{*}(t), S_{m}^{*}(0), 0\right)$.

Define $W^{s}\left(M_{1}\right)=\left\{x_{0}: \mathcal{P}^{n}\left(x_{0}\right) \rightarrow M_{1}, n \rightarrow \infty\right\}$, which is said to be a stable set of $M_{1}$. Since $\mathcal{R}_{0}>1$, the stable set $W^{s}\left(M_{1}\right)$ of $M_{1}$ satisfies that $W^{s}\left(M_{1}\right) \cap \mathbb{X}_{0}=\emptyset$. It follows from Lemmas 1 and 2 that $\left\{M_{1}\right\}$ is globally attractive in $M_{\partial}$ when $\phi_{2} b_{2}>\mu_{m 1}\left(\phi_{1}+\mu_{a 1}\right)$ and $\liminf _{t \rightarrow \infty} \Theta(t)>0$. Therefore, $\left\{M_{1}\right\}$ is isolated in $M_{\partial}$, and hence in $\mathbb{X}$. Furthermore, $\left\{M_{1}\right\}$ is also invariant and $\left\{M_{1}\right\}$ does not form a cycle in $M_{\partial}$, and hence in $\mathbb{X}$. By Theorem 1.3.1 in [34], we finally obtain that $\mathcal{P}$ is uniformly persistent with respect to $\left(\mathbb{X}_{0}, \partial \mathbb{X}_{0}\right)$, which implies that model (1) is uniformly persistent. The proof is completed.

As a consequence of Theorem 4, we have the following result.

Corollary 1 If $\mathcal{R}_{0}>1, \phi_{2} b_{2}>\mu_{m 1}\left(\phi_{1}+\mu_{a 1}\right)$ and $\liminf _{t \rightarrow \infty} \Theta(t)>0$, then model (1) possesses at least a positive $\omega$-periodic solution.

Particularly, when model (1) degenerates into the autonomous model with $\beta(t)=\beta$, $b(t)=b, k(t)=k, \phi(t)=\phi, \mu_{a}(t)=\mu_{a}, \alpha(t)=\alpha$, and $\mu_{m}(t)=\mu_{m}$ for all $t \geq 0$, it is easy to calculate that the autonomous model always has a disease-free equilibrium $E_{01}=$ $\left(\Lambda_{h} / \mu_{h}, 0,0,0,0,0\right)$. This implies that the mosquito population is eliminated. Notice that if $b \phi>\mu_{m}\left(\phi+\mu_{a}\right)$, then the autonomous model has one more disease-free equilibrium $E_{02}=\left(\Lambda_{h} / \mu_{h}, 0,0, A^{*}, S_{m}^{*}, 0\right)$, where

$$
A^{*}=\frac{k\left(b \phi-\mu_{m} \phi-\mu_{m} \mu_{a}\right)}{b \phi}, \quad S_{m}^{*}=\frac{k\left(b \phi-\mu_{m} \phi-\mu_{m} \mu_{a}\right)}{b \mu_{m}} .
$$


In this case,

$$
F=\left(\begin{array}{cc}
0 & \beta \Lambda_{h} / \mu_{h} \\
\alpha S_{m}^{*} & 0
\end{array}\right) \text { and } V=\left(\begin{array}{cc}
\gamma_{h}+\mu_{h}+d_{h} & 0 \\
0 & \mu_{m}
\end{array}\right)
$$

Using the method given in Ref. [25] (see also Lemma 2.2(ii) in Ref. [30]), we obtain the basic reproduction number of the autonomous model as follows:

$$
\begin{aligned}
\widetilde{\mathcal{R}}_{0} & =\sqrt{\frac{\Lambda_{h}}{\mu_{h}} \cdot \frac{\beta}{\gamma_{h}+\mu_{h}+d_{h}} \cdot \frac{\alpha}{\mu_{m}} \cdot \frac{k\left(b \phi-\mu_{m} \phi-\mu_{m} \mu_{a}\right)}{b \mu_{m}}} \\
& =\sqrt{\frac{\alpha \beta \Lambda_{h} k\left(b \phi-\mu_{m} \phi-\mu_{m} \mu_{a}\right)}{b \mu_{h} \mu_{m}^{2}\left(\gamma_{h}+\mu_{h}+d_{h}\right)}} .
\end{aligned}
$$

From the above expressions, we can see that $\alpha, \beta, k$, and $\phi$ are proportional to $\widetilde{\mathcal{R}}_{0}$, while $\mu_{m}$ and $\mu_{a}$ are inversely proportional to $\widetilde{\mathcal{R}}_{0}$. Using the sensitiveness analytical method [18], it is easily obtained that $\mu_{m}$ is the most sensitive parameter about the basic reproduction number $\widetilde{\mathcal{R}}_{0}$, followed by $\alpha, \beta, k$ and $\phi, b$, and $\mu_{a}$ are the least. Therefore, in order to effectively control the spread of the disease, during the rainy season, we can spray adult and larval insecticides, reduce contact rates, and reduce mosquito breeding sites at the same time. On the other hand, in view of pesticides causing environmental pollution, we can avoid the use of pesticides as much as possible in the dry season and control the spread of disease by reducing contact rates and reducing mosquito breeding sites.

\section{Numerical simulation and discussion}

In this section, some numerical simulations are presented to confirm the above theoretical analysis. Moreover, in order to illustrate the effect of seasonal succession on the dynamics of mosquito-borne disease, some numerical simulations are carried out. To this end, we choose some parameter values from Ref. $[19,36]$ and fix these parameters values as follows: $\Lambda_{h}=4, \eta=0.004, \mu_{h}=1 /(70 \times 365), \gamma_{h}=0.07, d_{h}=3.0 \times 10^{-6}, k_{1}=6.0 \times 10^{5}$, $k_{2}=1.5 \times 10^{4}, b_{1}=4, b_{2}=3 \phi_{1}=0.06, \phi_{2}=0.04, \mu_{a 1}=0.04, \mu_{a 2}=0.02 \mu_{m 1}=0.1$, and $\mu_{m 2}=0.07$.

For model (1), we choose, firstly, rainy season parameters $\beta_{1}=5.15 \times 10^{-7}, \alpha_{1}=$ $5.65 \times 10^{-7}$ and dry season ones $\beta_{2}=1.75 \times 10^{-7}, \alpha_{2}=1.88 \times 10^{-7}$. Clearly, $\phi_{2} b_{2}>$ $\mu_{m 1}\left(\phi_{1}+\mu_{a 1}\right)$, and $\liminf _{t \rightarrow \infty} \Theta(t)>0$ by numerical calculations. Further, the basic reproduction number is $\mathcal{R}_{0} \approx 0.9177<1$. Therefore, by Theorem 3 , the disease-free solution $\left(\Lambda_{h} / \mu_{h}, 0,0, A^{*}(t), S_{m}^{*}(t), 0\right)$ of model (1) is globally asymptotically stable. The corresponding numerical simulations are given in Fig. 1(a)-(c), in which the blue lines and red lines represent the solution curves of model (1) in the dry and rainy seasons, respectively. Numerical simulations show that the quantities of the infectious human and the infectious mosquitoes tend to zero from different initial values, which means that the disease is eliminated from this area eventually.

In order to investigate the effect of the seasonal succession on the disease-free periodic solution of model (1), we choose parameters $\beta=2.15 \times 10^{-7}, \alpha=4.65 \times 10^{-7}$, i.e., there is no seasonal variation. By direct calculation, we obtain the basic reproduction number $\widetilde{\mathcal{R}}_{0} \approx 0.9143$ of the autonomous model, which is close to the basic reproduction number $\mathcal{R}_{0} \approx 0.9177<1$ of model (1) with rainy season and dry season. The solution curves of two 
(a)
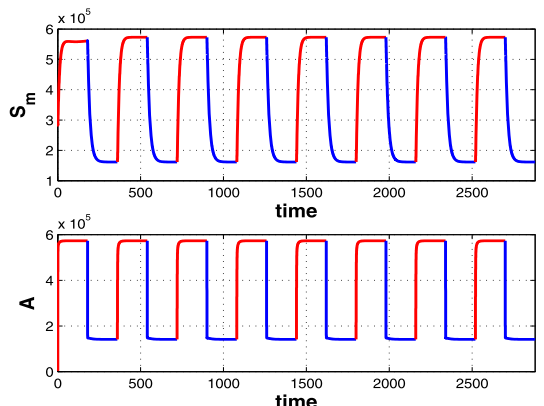

(c)

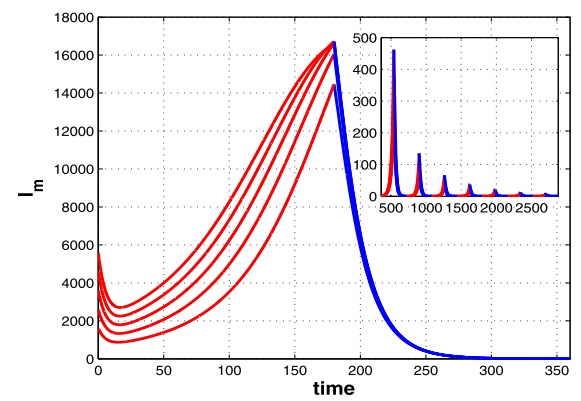

(b)

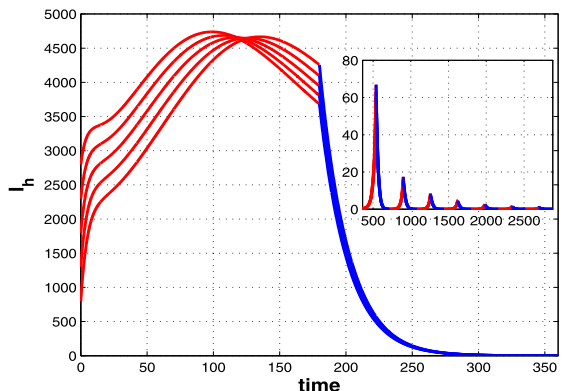

(d)

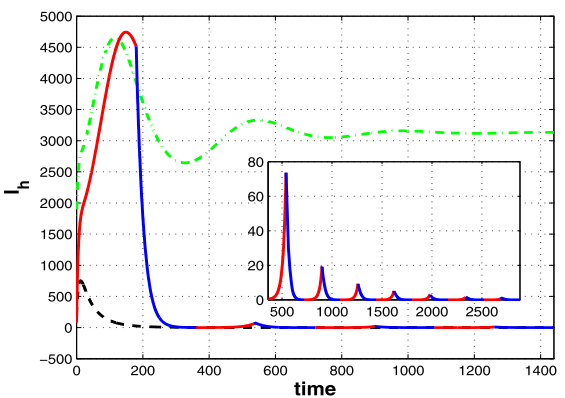

Figure 1 The global dynamics of disease-free solution of model (1) when $\mathcal{R}_{0}<1$

models are denoted by black dotted line and red-blue solid line in Fig. 1(d), respectively. By contrast of the trend of the black dotted line and the red-blue solid line, we find that the black dotted line undergoes a little bit of growth and then goes down very quickly and goes to zero; while the red-blue solid line has a relatively long growth and then gradually approaches zero after several disease cycles. These results indicate that even when the basic reproduction numbers are close to each other, it is easy to cause misjudgment for disease control departments without considering the seasonal succession. At the same time, we choose the above rainy season parameters for the autonomous model: $\beta=5.15 \times$ $10^{-7}, \alpha=5.65 \times 10^{-7}$, i.e., there is no seasonal variation. The simulation results are denoted by green chain dotted line in Fig. 1(d). Comparing the seasonal succession of rainy and dry, we find the basic reproduction number $\widetilde{\mathcal{R}}_{0} \approx 1.5597$ and the disease will not be extinct. That is to say, the disease will be overestimated without considering the dry season. The numerical simulation implies that considering the mosquito-borne epidemic model with seasonal change can more accurately describe the epidemic law of the mosquito-borne disease.

Furthermore, we choose rainy season parameters $\beta_{1}=1.35 \times 10^{-6}, \alpha_{1}=2.15 \times 10^{-6}$ and dry season parameters $\beta_{2}=5.5 \times 10^{-7}, \alpha_{2}=8.75 \times 10^{-7}$ in model (1). Clearly, $\phi_{2} b_{2}>$ $\mu_{m 1}\left(\phi_{1}+\mu_{a 1}\right)$, and by numerical calculations, one has $\liminf _{t \rightarrow \infty} \Theta(t)>0$ and obtains the basic reproduction number $\mathcal{R}_{0} \approx 2.2678>1$. According to Theorem 4 , model (1) is uniformly persistent, as shown in Fig. 2. In addition, the plots in Fig. 2(a) and Fig. 2(b) show that model (1) has a positive periodic solution, which is in accordance with the obtained conclusion in Corollary 1 . It seems that this positive periodic solution is globally attractive from the trend of solution curves with different initial values in Fig. 2(c) and Fig. 2(d). 
(a)

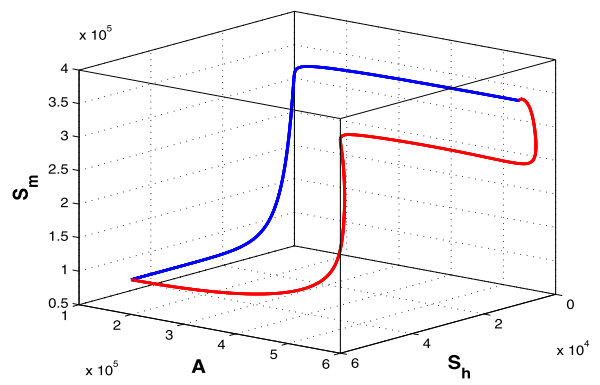

(c)

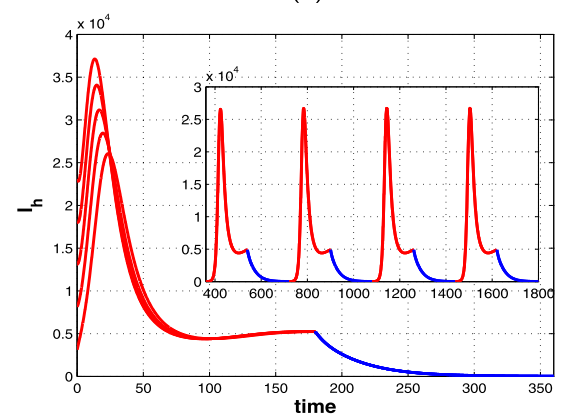

(b)

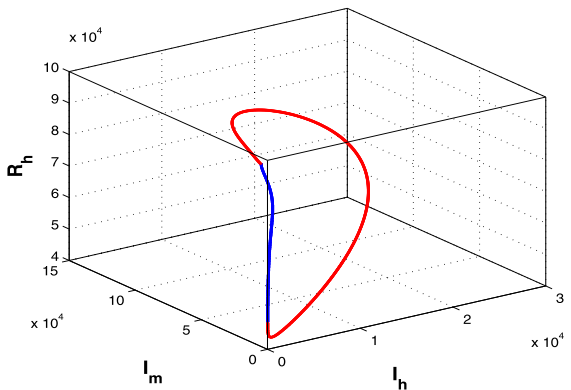

(d)

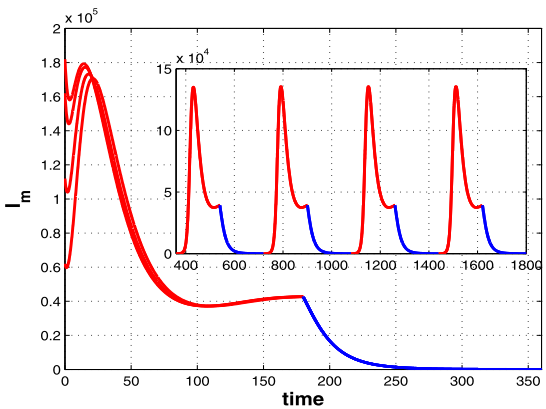

Figure 2 The uniform persistence of disease in model (1) and the existence of positive periodic solution of model (1) when $\mathcal{R}_{0}>1$ : (a) and (b) the existence of positive periodic solution; (c) and (d) the persistence and stability of this periodic solution

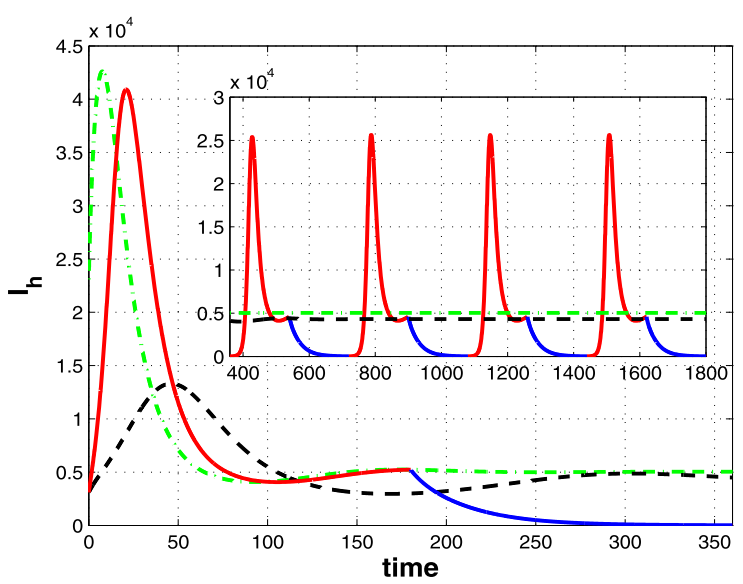

Figure 3 The effect of seasonal succession on the persistence of mosquito-borne disease

Finally, in order to investigate the effect of seasonal succession on the persistence of mosquito-borne disease, we take the autonomous model parameters $\beta=8.25 \times 10^{-7}$, $\alpha=1.23 \times 10^{-6}$, i.e., there is no seasonal succession. By calculating, we obtain the basic reproduction number $\widetilde{\mathcal{R}}_{0} \approx 2.2784$, which is close to the basic reproduction number $\mathcal{R}_{0} \approx 2.2678>1$ in Fig. 2 , where the seasonal succession is considered. This is shown in the black dotted line and the red-blue solid line in Fig. 3. It is not hard to find that the black dotted line undergoes a little bit of growth and then gradually goes to one value, while the 
red-blue solid line has a relatively big growth and then gradually forms a stable state of periodic fluctuation. Numerical simulations indicate that although the basic regeneration numbers of the two cases are very close, the prevalence of the disease is completely different. Further, we take only the rainy season parameters $\beta=1.35 \times 10^{-6}, \alpha=2.15 \times 10^{-6}$ in the autonomous model and get $\widetilde{\mathcal{R}}_{0} \approx 3.8224$ in this case. A similar result can be obtained, as shown by the green chain dotted line in Fig. 3. Theoretical results and numerical simulations show that neglecting the effect of seasonality on mosquitoes can lead to overestimating or underestimating the epidemic trend of mosquito-borne infectious diseases and cause waste of medical resources to a certain extent.

\section{Conclusion}

In this paper, we establish a mosquito-borne disease transmission model with seasonal succession, which is different from the existing work due to the discussion of the dynamics of transmission of mosquito-borne diseases in certain areas. These areas have a tropical monsoon climate, year-round high temperatures (suitable for mosquito growth), and only two seasons a year: the dry season and the rainy season. The rainfall difference between the two seasons is very obvious. Therefore, we use piecewise constant functions to describe the seasonal succession rather than the continuous periodic function. All in all, we can conclude our work and findings in the following two aspects:

(a) The main results obtained in this work: combined with the analysis methods in Refs. [30] and [23], the existence and uniqueness of the disease-free periodic solutions of this model are discussed, and the basic regeneration number of our model is obtained. On the premise of the existence of disease-free periodic solution, the basic regeneration number is the threshold that determines the extinction and persistence of the disease. Specifically, if $\mathcal{R}_{0}<1$, then the disease-free periodic solution of this model is globally asymptotically stable; if $\mathcal{R}_{0}>1$, the disease in the model is persistent and the model has a positive periodic solution.

(b) The effect of seasonal succession on the dynamics of the disease: in order to investigate the effect of seasonal succession on the dynamics of mosquito-borne disease, we give two other simulations to illustrate the effect of seasonal succession on the disease-free periodic solution and the persistence of the disease. These are shown in Fig. 1(d) and Fig. 3, respectively. Numerical simulations show that regardless of whether the disease is extinct or persistent, ignoring the effects of seasonal transitions, especially the cycle alternation of the dry and rainy seasons, on the total population of mosquito populations and their behavior inevitably overestimates or underestimates the disease trend. Therefore, the establishment of a reasonable seasonal mosquito-borne disease model to accurately predict the epidemic trend of the disease has very important guiding significance for the reasonable allocation and use of limited medical resources.

\section{Acknowledgements}

We would like to thank the anonymous referees for their helpful comments and the editor for his constructive suggestions, which have greatly improved the presentation of this paper. 
Availability of data and materials

Data sharing is not applicable to this article as no data sets were generated or analysed during the current study.

\section{Competing interests}

The authors declare that they have no competing interests.

\section{Authors' contributions}

Research design: all authors; Modelling: TZ and LN; Model analysis: TZ, ZT, and LN; Simulations: TZ and YL. All authors read and approved the final manuscript.

\section{Publisher's Note}

Springer Nature remains neutral with regard to jurisdictional claims in published maps and institutional affiliations.

Received: 14 April 2020 Accepted: 29 June 2020 Published online: 04 September 2020

\section{References}

1. Agusto, F.B.: Malaria drug resistance: the impact of human movement and spatial heterogeneity. Bull. Math. Biol. 76, 1607-1641 (2014)

2. Altizer, S., Dobson, A., Hosseini, P., et al.: Seasonality and the dynamics of infectious diseases. Ecol. Lett. 9, 467-484 (2006)

3. Amaku, M., Raimundo, S.M., Lopez, L.F., et al.: A comparative analysis of the relative efficacy of vector-control strategies against dengue fever. Bull. Math. Biol. 76, 697-717 (2014)

4. Andraud, M., Hens, N., Beutels, P.: A simple periodic-forced model for dengue fitted to incidence data in Singapore. Math. Biosci. 244, 22-28 (2013)

5. Aron, J.L.: Mathematical modelling of immunity to malaria. Math. Biosci. 90, 385-396 (1988)

6. Bainov, D., Simeonov, P.: Impulsive Differential Equations: Periodic Solutions and Applications. Longman, New York (1993)

7. Bowman, C., Gumel, A.B., van den Driessche, P., et al.: A mathematical model for assessing control strategies against West Nile virus. Bull. Math. Biol. 67, 1107-1133 (2005)

8. Chiyaka, C., Tchenche, J.M., Tchuenche, W., et al.: A mathematical analysis of the effects of control strategies on the transmission dynamics of malaria. Appl. Math. Comput. 195, 641-662 (2008)

9. Cruz-Pacheco, G., Esteva, L., Vargas, C.: Seasonality and outbreaks in West Nile virus infection. Bull. Math. Biol. 71 1378-1393 (2009)

10. Cui, J.A., Chen, L.S., Wang, W.D.: The effect of dispersal on population growth with stage-structure. Comput. Math. Appl. 39, 91-102 (2000)

11. Erickson, R.A., Presley, S.M., Allen, L.J.S., et al.: A dengue model with a dynamic Aedes albopictus vector population. Ecol. Model. 221, 2899-2908 (2010)

12. Esteva, L., Yang, H.: Assessing the effects of temperature and dengue virus load on dengue transmission. J. Biol. Syst. 23, $1550027(2015)$

13. Gopalsamy, K.: Stability and Oscillations in Delay Different Equations of Population Dynamics. Kluwer Academic, Dordrecht (1992)

14. International Travel Health Advisory Network. http://www.ithc.cn/article/341855.html

15. Lou, Y., Zhao, X.Z:: A climate-based malaria transmission model with structured vector population. SIAM J. Appl. Math. 70, 2023-2044 (2010)

16. Malaria. https://www.who.int/zh/news-room/fact-sheets/detail/malaria

17. McLennan-Smith, T.A., Mercer, G.N.: Complex behaviour in a dengue model with a seasonally varying vector population. Math. Biosci. 248, 22-30 (2014)

18. Nakul, C., James, M.H., Jim, M.C.: Determining important parameters in the spread of malaria through the sensitivity analysis of a mathematical model. Bull. Math. Biol. 70, 1272-1296 (2008)

19. Pinho, S.T.R., Ferreira, C.P., Esteva, L.: Modelling the dynamics of dengue real epidemics. Philos. Trans. R. Soc. Lond. A 368, 5679-5693 (2010)

20. Rodrigues, H.S., Monteiro, M.T.T., Torres, D.F.M.: Seasonality effects on dengue: basic reproduction number, sensitivity analysis and optimal control. Math. Methods Appl. Sci. 39, 4671-4679 (2016)

21. Ross, R.: The Prevention of Malaria, 2nd edn. Murray, London (1910)

22. Ruan, S.G., Xiao, D.M., Beier, J.C.: On the delayed Ross-Macdonald model for malaria transmission. Bull. Math. Biol. 70, $1098-1114(2008)$

23. Tang, Y.L., Xiao, D.M., Zhang, W.N., Zhu, D.: Dynamics of epidemic models with asymptomatic infection and seasonal succession. Math. Biosci. Eng. 14, 1407-1424 (2017)

24. Tumwiine, J., Mugisha, J.Y.T., Luboobi, L.S.: A mathematical model for the dynamics of malaria in a human host and mosquito vector with temporary immunity. Appl. Math. Comput. 189, 1953-1965 (2007)

25. Van Den Driessche, P., Watmough, J.: Reproduction numbers and sub-threshold endemic equilibria for compartmental models of disease transmission. Math. Biosci. 180, 29-48 (2002)

26. Vargas-De-León, C.: Global analysis of a delayed vector-bias model for malaria transmission with incubation period in mosquitoes. Math. Biosci. Eng. 9, 165-174 (2013)

27. Vargas-De-León, C., Esteva, L., Korobeinikov, A.: Age-dependency in host-vector models: the global analysis. Appl. Math. Comput. 243, 969-981 (2014)

28. Wang, L., Teng, Z.D., Zhang, T.L.: Threshold dynamics of a malaria transmission model in periodic environment. Commun. Nonlinear Sci. Numer. Simul. 18, 1288-1303 (2013)

29. Wang, X., Chen, Y.M., Liu, S.Q.: Dynamics of an age-structured host-vector model for malaria transmission. Math. Methods Appl. Sci. 41, 1966-1987 (2018) 
30. Wang, X.N., Zhao, X.Q.: Threshold dynamics for compartmental epidemic models in periodic environments. J. Dyn. Differ. Equ. 20, 699-717 (2008)

31. Wang, X.N., Zhao, X.Q.: A periodic vector-bias malaria model with incubation period. SIAM J. Appl. Math. 77, 181-201 (2017)

32. Wei, H.M., Li, X.Z., Martcheva, M.: An epidemic model of a vector-borne disease with direct transmission and time delay. J. Math. Anal. Appl. 342, 895-908 (2008)

33. Wu, R., Zhao, X.Q.: A reaction-diffusion model of vector-borne disease with periodic delays. J. Nonlinear Sci. 29, 29-64 (2019)

34. Xu, Z.T., Zhao, Y.Y: A diffusive dengue disease model with nonlocal delayed transmission. Appl. Math. Comput. 270 808-829 (2015)

35. Yang, H.M., Ferreira, C.P.: Assessing the effects of vector control on dengue transmission. Appl. Math. Comput. 198, 401-413 (2008)

36. Zou, L., Chen, J., Feng, X.M., Ruan, S.G.: Analysis of a dengue model with vertical transmission and application to the 2014 dengue outbreak in Guangdong province. Chin. Bull. Math. Biol. 80, 2633-2651 (2018)

\section{Submit your manuscript to a SpringerOpen ${ }^{\circ}$} journal and benefit from:

- Convenient online submission

- Rigorous peer review

- Open access: articles freely available online

- High visibility within the field

- Retaining the copyright to your article

Submit your next manuscript at $\boldsymbol{~ s p r i n g e r o p e n . c o m ~}$ 